\title{
CONOCIMIENTOS Y SABERES LOCALES EN TRES PROPUESTAS CURRICULARES PARA EDUCACIÓN INDÍGENA
}

\section{LOCAL KNOWLEDGE IN THREE CURRICULAR PROPOSALS FOR INDIGENOUS EDUCATION}

\section{Rosa María Salgado Medina rossal65@gmail.com}

Currículo: maestría en Educación. Profesora-investigadora de la Universidad Pedagógica Nacional. Sus líneas de investigación versan sobre la interculturalidad, el currículo, la educación en línea y las relaciones de poder.

\section{Ulrike Keyser Ohrt}

ulrikekeyser@upn162-zamora.edu.mx

Currículo: doctora en Educación. Profesora-investigadora de la Universidad Pedagógica Nacional. Sus líneas de investigación versan sobre la educación intercultural e indígena, la formación de docentes y el aprendizaje colaborativo.

\section{Gabriela Ruiz de La Torre} upn161@yahoo.com.mx

Currículo: doctora en Derecho. Profesora-investigadora de la Universidad Pedagógica Nacional. Sus líneas de investigación versan sobre el currículo, los derechos humanos, la educación y la sustentabilidad.

Recibido: 25 de agosto de 2017. Aceptado para su publicación: 15 de enero de 2018. Recuperado de: https://sinectica.iteso.mx/index.php/SINECTICA/article/view/782 DOI: 10.31391/S2007-7033(2018)0050-003

\section{Resumen}

En este trabajo explicamos la relevancia de los conocimientos locales e indígenas y su incorporación en procesos educativos; ambas son condiciones para la transformación económica, social, política y educativa que requiere el México pluriétnico y la construcción de relaciones más igualitarias. Saberes distintos al pensamiento occidental dominante empiezan a ser reconocidos dentro de la educación formal y esto ha permitido evaluar problemas, visualizar obstáculos, determinar retos y diseñar alternativas de acción docente. En este marco, revisamos tres propuestas: el programa de la licenciatura en Educación Preescolar y Primaria para el Medio Indígena, que imparte la Universidad Pedagógica Nacional; el método inductivo intercultural, que se ha trabajado con estudiantes y egresados de la licenciatura mencionada; y el Programa de Educación para los Pueblos Originarios de Michoacán, diseñado y aplicado por docentes indígenas a partir de los conocimientos y las prácticas locales en las comunidades. El proceso metodológico comprendió un estudio documental comparativo de los diseños curriculares, entrevistas y grupo de discusión con docentes de las tres propuestas. Los resultados subrayan la importancia de la participación activa e investigación continua del profesorado en las comunidades para una educación basada en conocimientos indígenas.

Palabras clave: conocimientos indígenas, programas de educación indígena e intercultural, formación docente. 


\begin{abstract}
In this work we explain the relevancy of the local and Indigenous knowledge and its incorporation in the educational processes; both, conditions for the economic, social, political and educational transformation that needs the multi-ethnic reality of Mexico and the establishment of a fairer system. In the dominant education system, other forms of knowledge are being recognized. This has allowed evaluating problems, visualizing obstacles, determining challenges and designing alternatives of teaching action. With this reference, the aim of the article is to analyze three proposals: the Degree in Preschool and Primary Education for the Indigenous Context, curriculum offered by the National Pedagogic University, the Intercultural Inductive Method that has worked with students who graduated from National Pedagogic University and the Educational Program for the Native Peoples of Michoacán, designed and applied by Indigenous teachers based on the knowledge and local practices of the communities. The methodological process included a comparative study of the curricular designs, interviews and discussion group with teachers of the three proposals. The results underline the importance of continuous research and active participation of teachers in the communities for an education based on Indigenous knowledge. Keywords: indigenous knowledge, indigenous and intercultural education programs, teacher training.
\end{abstract}

\title{
INTRODUCCIÓN
}

Sin el empoderamiento de los sistemas de conocimiento tradicionales y sin resolver los problemas estructurales, de subordinación, explotación y discriminación de sus portadores, el posible diálogo de saberes no es más que una escenografía para un proceso demagógico, folclorizante y expropiatorio

(Pérez y Argueta, 2011)

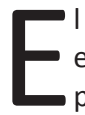

I interés por investigar y trabajar con los conocimientos locales dentro de la escuela se ha vuelto el eje principal de actividades pedagógicas y diseños de programas en el campo de la educación indígena. Esto implica la necesidad de formar al profesorado para que puedan asumir y cumplir esta tarea. En este contexto, presentamos y analizamos tres programas que tienen dos aspectos en común: por una parte, la formación docente y, por otra, son propuestas curriculares orientadas a transformar el quehacer cotidiano en las aulas.

En México, la Universidad Pedagógica Nacional (UPN) fue la primera institución formadora de maestros que diseñó un programa específico para docentes que laboraban en el medio indígena. Este programa corresponde a la licenciatura de Educación Preescolar y Primaria para el Medio Indígena (LEPEPMI), que se ofrece desde 1990 en unidades y subsedes distribuidas en diferentes entidades donde hay población indígena. El diseño e implementación de esta licenciatura es el primer programa que se analiza en este trabajo.

Como resultado de una evaluación nacional en 2007, el diseño curricular de este programa se reestructuró; sin embargo, los cambios se aplicaron únicamente en algunas de las sedes: se empezó por los estados de Chiapas, Puebla y Oaxaca, y después se integraron Michoacán y Yucatán. Esta reformulación curricular consistió en sustituir los cursos del séptimo y octavo semestres del plan de estudios por los diplomados Explicitación y Sistematización de Conocimientos Indígenas y 
Elaboración de Materiales Educativos Interculturales y Bilingües, ambos basados en el método inductivo intercultural (MII).

El método y los diplomados corresponden a la segunda propuesta que se analiza en este documento y son resultado de experiencias formativas, así como de un diseño curricular construido "desde abajo" (Bertely, 2006) con la colaboración de Jorge Gasché, del Instituto de Investigaciones de la Amazonia Peruana (IIAP), educadores de la Unión de Maestros de la Nueva Educación para México (UNEM) de Chiapas e investigadores del Centro de Investigaciones y Estudios Superiores en Antropología Social (CIESAS). En este programa de formación docente ocupan un lugar central los conocimientos indígenas, implícitos en las actividades productivas, sociales y rituales desarrolladas en las comunidades indígenas (Gasché, 2008); por lo anterior, las prácticas discursivas representan un eje de interpretación de las mismas actividades.

Por otra parte, la tercera propuesta que analizamos se vincula con el movimiento magisterial de resistencia y oposición al régimen, que tiene un gran número de simpatizantes en el estado de Michoacán. Parte de este movimiento fueron las luchas sindicales del verano de 2016 contra la Reforma Integral de Educación Básica y la Ley General del Servicio Profesional Docente, que dieron un impulso para que docentes sindicalizados, junto con autoridades y especialistas del sistema de educación indígena en el estado, tomaran la iniciativa de diseñar un proyecto propio: el Programa de Educación para los Pueblos Originarios de Michoacán (PEPOMICH), que se ha implementado en los niveles de educación inicial, preescolar y primaria desde el ciclo escolar 2016-2017 en centros escolares de comunidades indígenas p’urhépechas, nahuas, mazahuas y otomíes.

\section{UN PUNTO DE PARTIDA: LAS INQUIETUDES}

El propósito de este trabajo es compartir los resultados parciales de una investigación que problematiza la presencia y el uso didáctico de saberes y conocimientos indígenas desde la formación docente hasta su aplicación en el desarrollo curricular en educación básica. Reconocemos y analizamos tanto los aportes de cada una de las tres propuestas como las diferencias entre ellas; esto, a fin de dar cuenta de lo que pueden ofrecer a una educación que parte del reconocimiento y desarrollo de los conocimientos indígenas y locales para contrarrestar el dominio de conocimientos llamados "universales". Las preguntas que guían la investigación son: ¿de qué manera se reconocen tanto los conocimientos como las prácticas indígenas y locales en el plan de estudios de la LEPEPMI y en las propuestas del MII y el PEPOMICH? y ¿qué tipo de conocimientos indígenas están presentes en este programa y en ambas propuestas?

Para contestar estas preguntas, nuestro diseño metodológico comprendió el uso de diversas fuentes. En primer lugar, mediante un estudio documental de los diseños curriculares, seleccionamos y comparamos los contenidos que refieren a conocimientos indígenas y locales en cada uno de los tres programas. Posteriormente, para conocer los resultados y experiencias en el aula, solicitamos el apoyo de docentes en servicio que tenían relación con la UPN a través de las unidades de Morelia, Zamora y Uruapan. En adición, las autoras hemos colaborado como profesoras en la LEPEPMI y en los diplomados con el MII; esto nos ha dado la oportunidad de dirigir tesis de maestría que recuperan el trabajo desarrollado con el PEPOMICH y conocer de modo directo el sentir, los problemas, los retos y las alternativas de los docentes frente a grupo. 
De manera particular, entrevistamos a dos coordinadores de la LEPEPMI con la finalidad de recuperar información relacionada con la formación de docentes en contextos indígenas. Organizamos un grupo de discusión con cinco docentes que trabajan con el PEPOMICH en el nivel inicial, preescolar y primaria, y que formaron parte de los equipos de diseño. Las experiencias de formación y práctica docente del MII se recuperaron desde los diarios de campo elaborados con los resultados de las observaciones de cada sesión de trabajo de las tres generaciones de diplomantes que egresaron en el estado y en reuniones de trabajo con un equipo de educadoras en la comunidad de Cherán, Michoacán.

\section{CONOCIMIENTOS Y SABERES LOCALES E INDÍGENAS}

En una educación que se considera intercultural, tanto los conocimientos propios que han desarrollado las culturas que conforman el contexto comunitario de los docentes y sus educandos como las lenguas originarias o maternas que las expresan y transmiten tendrán que estar presentes y ser utilizadas en la escuela de manera que ninguna se deseche. Este planteamiento pedagógico considera que es a partir de lo propio que se puede conocer y valorar lo ajeno. En palabras de Lacán (2016), diríamos que el reconocimiento del yo es el primer paso, después se reconoce y valora al Otro.

Sin embargo, en el reconocimiento de ese yo primario existe un vacío (Žižek, citado en Castro-Gómez, 2015) que impide al sujeto el goce pleno de sus experiencias y de ahí la enajenación o negación de lo propio; esto último obstruye un desarrollo integral y continuo en los alumnos y contraviene a su formación como sujetos autónomos, conscientes y participativos en una sociedad y cultura cambiantes. Para que esta sociedad y cultura respondan a su propia necesidad -y no solo a las de quienes dominan económica y políticamente-, se deberá partir de la construcción y valoración de una identidad propia. Por lo anterior, en el caso de este estudio, interesa resaltar la diferencia entre saberes propios y aquellos que la ideología dominante occidental, como hegemónica, ha impuesto.

La reducción del concepto de conocimiento a conocimientos legitimados por la ciencia de cuño europeo es expresión de las relaciones de poder que existen no únicamente en el ámbito económico y político en el mundo actual, sino que da cuenta que también el conocimiento es poder. Al respecto, Foucault (2014) señala que el sujeto existe solo en relación con el saber y el poder; en función de esta idea, se comprende el interés por justificar ciertos conocimientos como relevantes y utilizarlos para mantener un lugar privilegiado dentro de la estructura económica y social.

Además, visto de este modo, el conocimiento impuesto desde las ciencias formales y las empíricas es, a la luz del análisis de Galtung (2003), una de las manifestaciones de la violencia cultural. En este sentido, los conocimientos indígenas forman parte de los conocimientos reprimidos y excluidos de los que tomaron el liderazgo científico de carácter eurocéntrico y que presume ser universal; “... el discurso de la modernidad creó la ilusión de que el conocimiento es abstracto, des-incorporado y des-localizado, haciéndonos pensar que es universal, que no tiene casa o cuerpo, ni tampoco género o color" (Walsh, 2006, p. 19). Lo que sucede en realidad es que los conocimientos se generan, modifican y practican en determinados espacios y tiempos. 
Los conocimientos indígenas que forman parte del ethos se transmiten de manera oral y se manifiestan en las prácticas productivas, sociales y rituales que realiza la población de una comunidad indígena; a decir de Gramsci (2013), son parte de la misma ideología que les ata, del saber occidental que ha condicionado al Ser. Para distinguir estos conocimientos locales, analizar su transformación y recuperarlos desde la escuela, tendrán que explicarse y escuchar todas las voces; en caso contrario, se cometería de la misma manera el uso del poder para imponer o legitimar saberes que corresponden a los grupos dominantes de ese sector. En este sentido, resulta indispensable recuperar los resultados de las investigaciones realizadas por los docentes de esos contextos, quienes registran el modo en que dichos conocimientos son utilizados para construir nuevos saberes.

Con el propósito de definir lo que se entiende por conocimientos indígenas, en la II Cumbre Continental de los Pueblos y Nacionalidades Indígenas de Abya Yala (2004) se recuperaron las perspectivas de distintas etnias del continente americano; ahí se consideró:

-Que, el conocimiento indígena es un conjunto integrado de Saberes y Vivencias de las culturas fundamentadas en nuestras experiencias, praxis milenaria y su proceso de inter-acción permanente hombre-naturaleza y la divinidad;

-Que, las sabidurías ancestrales y el conocimiento colectivo tienen un vínculo imprescindible con las tierras y territorios, y la existencia como Pueblos Indígenas;

- Que, los sistemas de propiedad intelectual no son una institución que deba contemplar disposiciones acerca de los saberes ancestrales y conocimientos colectivos de los Pueblos Indígenas;

-Que, los Pueblos Indígenas somos sujetos de nuestra propia autonomía y libre determinación;

-Que, los conocimientos Indígenas deben ser revitalizados, fortalecidos y aplicados;

-Que, los conocimientos Indígenas son colectivos y comunitarios, por lo tanto deben ser respetados por los Gobiernos y otros actores externos;

-Que, los conocimientos Indígenas están centrados en la Madre Naturaleza, Pachamama (es holístico); y,

-Que, los conocimientos Indígenas son inviolables, inalienables e imprescriptibles y son de carácter intergeneracional (tema 4).

En el pronunciamiento anterior se destaca el carácter colectivo de los conocimientos indígenas y su fundamento en las prácticas e interacciones entre humanos y naturaleza. Si se pretende fortalecer este tipo de conocimiento mediante la educación escolarizada, es necesario, además de registrarlos, crear las condiciones culturales, económicas, sociales y estructurales para que el profesorado, junto con las niñas y los niños, puedan practicarlos dentro y fuera de la escuela, en coordinación con personas mayores de la comunidad; esto permitirá procesos de enseñanza y aprendizaje intergeneracionales:

Situados en el tiempo y en el espacio, "los saberes populares se mantienen en las prácticas técnicas y también sociales y su eficacia depende de las relaciones entre las personas que participan" (Friedberg). No se trata de saberes estáticos, sino de saberes que se construyen según las modificaciones del contexto. [...] el conocimiento indígena es holístico, subjetivo y experiencial (Quilaqueo, 2007, p. 227). 
Esto implica que el trabajo educativo con conocimientos indígenas desde la escuela se tiene que realizar a través de actividades prácticas, de preferencia fuera del aula, para articular el contexto comunitario con el escolar. Se requieren estructuras que rompan la dicotomía entre comunidad y escuela, que permitan procesos comunicativos en los que ambas partes puedan aprender mutuamente y se basen en la reflexión de los procesos históricos que han obstaculizado la igualdad en las relaciones entre ambos espacios.

Con regularidad, los objetivos de la escuela se ven como opuestos o sin ninguna relación con los intereses o problemas que enfrenta la comunidad. Esta situación es el resultado de dos perspectivas contradictorias que conviven y surgen como propuestas educativas desde el Estado. La primera surge en la época de Vasconcelos; con base en esta se han diseñado programas para formar el tipo de indígena que se ajusta a las necesidades del Estado. El resultado ha sido la estabilidad, el reconocimiento y la consolidación de una cultura homogénea a expensas de la negación del yo indígena, desde sí mismo y desde el Otro.

De la segunda perspectiva surge una educación cuyo interés principal es la consolidación de una identidad indígena, que reivindica el uso de las lenguas originarias (educación bilingüe-bicultural), así como en su versión intercultural, y hace hincapié en la importancia del reconocimiento de la diversidad cultural y lingüística para que las culturas se enriquezcan mutuamente, como lo postula la Coordinación General de Educación Intercultural y Bilingüe (Dietz y Mateos, 2011, p. 11).

A esta educación para indígenas se contrapone otra de indígenas que se practica principalmente en las familias y comunidades, fuera de la escuela. En las tres propuestas estudiadas observamos que son amplias las posibilidades del docente dentro del aula para reconstruir un tipo de saber, utilizar métodos didácticos alternativos adecuados al pensamiento comunitario y romper con la dicotomía escuela-comunidad al definir nuevas funciones para el aprendizaje y la enseñanza.

Sin embargo, reconocemos que el trabajo educativo con conocimientos indígenas implica una propuesta político-pedagógica alternativa a la del sistema educativo oficial y, por ello, requiere el trabajo tanto conjunto como consensuado de todos los sectores de la sociedad.

La educación intercultural busca la inclusión de otras culturas y lenguas, pero no se limita a ello o al enriquecimiento que puede derivar del contacto entre diferentes culturas, sino que pone en el centro las relaciones asimétricas de poder entre las culturas en contacto y coloca a los conocimientos en un lugar central junto con las distintas maneras en que se construyen. Además, se dirige hacia la descolonización de los conocimientos y se fundamenta en un diálogo de saberes entre pares para crear las bases de una socialización igualitaria entre quienes pertenecen a distintas culturas y habitan un mismo país:

... el sentido de la educación es justamente convertir a los individuos en sujetos pertinentes del saber [sujetos que tienen acceso a ciertos saberes y, por lo tanto, pueden juzgarlos]; su meta es lograr que la mayoría de los hombres no quede excluida de las comunidades epistémicas de la sociedad a que pertenecen. Así, la educación es el proceso para adecuar las comunidades epistémicas a la sociedad real (Villoro, 2008, pp. 148-149). 
Con base en las consideraciones sobre conocimientos indígenas y locales, así como en las premisas para un diálogo de saberes en condiciones de igualdad, presentamos a continuación los análisis curriculares del programa de la LEPEPMI y las propuestas alternativas del MII y del PEPOMICH.

Por análisis curricular entendemos la descripción de los elementos que componen el diseño de planes y programas: esto permite determinar la manera en que se reconocen los saberes indígenas en los documentos respectivos.

\section{la licenciatura en Educación Preescolar y Educación Primaria para el MEDIO INdíGENA (LEPEPMI)}

Consideramos importante incluir en nuestro análisis el plan de estudios que ofrece la UPN para docentes del medio indígena, ya que, de 2,030 docentes, 1,238 se han formado con base en él, es decir, $60.98 \%$ de quienes atendieron los grupos de educación inicial, preescolar y primaria en Michoacán durante el ciclo escolar 2016-2017. Aunque desde el ciclo escolar 2004-2005 se reconocen oficialmente los estudios de la Escuela Normal Indígena de Michoacán (ENIM), sus egresados todavía son una minoría, pues solo representan $17.88 \%$, esto es, 363 de 2,030 docentes de educación indígena en esta entidad (Dirección de Educación Indígena, 2017); el resto (21.14\%) egresaron de otras instituciones y programas.

La LEPEPMI, que se trabaja en la modalidad semiescolarizada en las unidades UPN de 23 estados de la república (Galicia, citado en Von Groll, Keyser y Silva, 2013, p. 162), tiene como antecedente la licenciatura en Educación Indígena que la Unidad Ajusco ofrece desde 1982 en forma escolarizada. El propósito de esta licenciatura -la primera a nivel superior especializada en la formación de docentes indígenas- es transformar la práctica y esto "ha sido la razón de ser del proyecto desde su inicio" (Reyes, 2016, p. 118).

En la LEPEPMI se forman profesionales de la educación capaces de fortalecer y rescatar la cultura de las comunidades indígenas, especialmente aquello que se relaciona con el aprendizaje de la lengua p'urhépecha y los procesos sociales en las comunidades. Por lo anterior, los docentes, formadores de estos profesionales, definen la educación intercultural como aquella que permite la coexistencia e inclusión de distintas manifestaciones culturales. De ahí que se le considera una educación para todos, basada en un modelo educativo que permite la construcción de una identidad, no solo indígena, lo que coadyuva tanto al desarrollo integral como a la consolidación de una convivencia más igualitaria.

El plan de estudios de la LEPEPMI (o LEP y LEPMI '90) (UPN, s.f., 2002, 2016a y b) consta de ocho semestres y su mapa curricular abarca dos áreas: básica y terminal. El área básica consta de cuatro líneas: psicopedagógica, sociohistórica, antropológica lingüística y metodológica. El área terminal incluye los siguientes campos desarrollados a partir del sexto semestre del plan de estudios: la naturaleza, lo social, la lengua y la matemática. Como podemos observar, tanto las líneas como los campos refieren divisiones entre las ciencias a partir de la postura del pensamiento occidental. Sus diferentes programas facilitan el registro de conocimientos en diversos campos; en lo particular, se destacan, por una parte, las características socioeconómicas y las particularidades lingüísticas y culturales del alumnado y, por otra, las formas de comunicación y de organización social del trabajo, tanto en la familia como en la comunidad.

Los saberes incluyen conocimiento de los estilos y prácticas culturales, explica- 
ciones propias de los fenómenos naturales y experiencias expresadas por todos; en el proceso de aprendizaje, estos se utilizan como andamios para la construcción de nuevos conocimientos científicos. Se pretende que quienes son parte del profesorado apliquen estrategias adecuadas para apoyar el desarrollo de la competencia comunicativa y lingüística a nivel oral de la lengua materna o en la segunda lengua con las referencias étnicas y culturales respectivas.

En el diseño curricular se sugiere abordar los conocimientos indígenas mediante registros de información en diversos campos, de manera que sirvan exclusivamente como mediadores para el aprendizaje de contenidos escolares. En este sentido, "lo indígena aparece en el contexto comunitario, pero no logran integrarse las prácticas o saberes comunitarios a la enseñanza como contenidos, ni como estrategias, tampoco se revisa su diferencia frente a lo que se pretende enseñar para, de esa manera, favorecer una educación intercultural" (Jordá, citado en Reyes, 2016, p. 117); es decir, los conocimientos o saberes son identificados por los docentes en formación, pero no se reflexiona sobre su importancia dentro de los procesos educativos escolares o como contenidos en sí mismos.

En este programa, la formación de los docentes se centra en asuntos de la lengua y la cultura (coordinador de LEPEPMI, 2017); con ello se pretende lograr una resignificación identitaria como indígenas. Para algunos docentes, el tratamiento de lo propio se centra en la lengua y la cultura, elementos que parecen ser lo mismo, como se refiere en la siguiente idea: "iLos maestros bilingües está[n] pensando que la lengua ya es sinónimo... ya es... toda la cultura, cuando sólo es un componente de la cultura... la lengua...!" (docente de la LEPEPMI, citado en Reyes, 2016, p. 118). No es intención de la LEPEPMI modificar las estructuras políticas ni construir alternativas al sistema educativo que son la base de las relaciones asimétricas de poder; no obstante, a quienes han egresado de este programa se les ha formado con las actitudes, habilidades y sentido crítico que permiten diseñar y aplicar otras vías de educación para y de indígenas.

Estas alternativas diseñadas se circunscriben a proponer cambios pedagógicos y didácticos dentro de los límites que marca la Secretaría de Educación Pública (SEP). Con el propósito de romper estos círculos, se promueven negociaciones, diálogos entre la parte sindical y agentes comunitarios, por un lado, y la oficial o autoridad, por el otro. En esos espacios se han trabajado modelos de educación alternativa, entendida como "una educación que está explícita y deliberadamente orientada a revertir el modelo social excluyente desde el que ha sido configurada la sociedad" (Méndez, citado en Bermúdez, Cuevas y Hernández, 2015, p. 364). Así también lo entienden docentes que han participado en un programa alternativo: "¿Entonces vamos a contrariar los programas oficiales?" A lo que le contestó otro: "Exactamente" (Keyser, 2011, p. 127). Quizá la respuesta no sea contrariar, sino analizar el sentido de la educación pública y definir el sentido de la educación indígena.

En función de lo dicho hasta el momento, afirmamos que la LEPEPMI constituye un primer paso en la formación de docentes; sin embargo, el trabajo continuo entre maestros y formadores de docentes o investigadores debe robustecerse con intercambios de experiencias permanentes, más allá de la propuesta que se presente para la obtención de un título.

PROPUESTAS ALTERNATIVAS A LOS PROGRAMAS OFICIALES PARA LA EDUCACIÓN BÁSICA Las propuestas alternativas que en este documento analizamos refieren el MIl y el $\mathrm{PEPOMICH}$. El primero se caracteriza por su origen interdisciplinario e interétnico; 
quienes apoyan su aplicación son especialistas con distinta formación. A partir de experiencias de formación de docentes indígenas en la Amazonia peruana y el reconocimiento de los conocimientos indígenas/locales en esta región, Jorge Gasché, lingüista y antropólogo, sentó las bases pedagógicas y políticas de este método. Posteriormente, en el contexto del levantamiento zapatista, esta propuesta fue aceptada y desarrollada en Chiapas por los integrantes tseltales, tsotsiles y ch'oles de la Unión de Maestros de la Nueva Educación para México y Educadores Independientes (UNEM/EI) con apoyo de María Bertely del CIESAS y otros investigadores del campo educativo.

El proceso de formación de docentes y educadores con el MII inició en 1995 (Bertely, 2006). Desde 2007 se han formado docentes del medio indígena en los estados de Chiapas, Puebla, Oaxaca, Michoacán y Yucatán por medio de los diplomados: Sistematización del Conocimiento Indígena y Diseño de Materiales Educativos Interculturales y Bilingües (Bertely y REDIIN, 2011). En un inicio los diplomados se trabajaron en sustitución de los programas del $7^{\circ}$ y $8^{\circ}$ semestres de la LEPEPMI; a la fecha se ofrecen a docentes del medio indígena en servicio como parte de su actualización y formación permanente.

La segunda propuesta que se presenta es el PEPOMICH. En comparación con el MII, en el cual, en congruencia con su concepto de interculturalidad, los diseñadores y capacitadores son tanto indígenas como no indígenas, el PEPOMICH es una iniciativa exclusivamente indígena entre la parte sindical indígena (Sector IX de la Sección XVIII, CNTE-SNTE) y la Dirección de Educación Indígena en el estado de Michoacán.

El diseño del PEPOMICH inició en el ciclo escolar 2014-2015. Está basado en el Proyecto para el Desarrollo Curricular Alternativo de Educación Indígena en Michoacán. De acuerdo con el secretario de Educación y Cultura del Sector IX, Quildo Santos, desde su presentación ante el secretario de Educación en el estado, en 2017, dicho proyecto recibió el nombre de Programa para la Educación de los Pueblos Originarios de Michoacán. Su objetivo es

\begin{abstract}
construir un plan y programas de estudio específicos para la educación de los pueblos originarios, [...] partir de las necesidades de aprendizaje de los estudiantes, crear contenidos de enseñanza propios, rescatando, investigando y preservando los saberes comunales, ampliando el conocimiento de y en las lenguas maternas (DGEI/Sector IX, 2016, p. 17) [...] para formar individuos capaces de enfrentar los retos actuales con aportes de sus saberes y los universales que coadyuven a su preservación y fortalecimiento (DGEI y Sector IX, 2016, p. 8).
\end{abstract}

Aunque el diseño del proyecto ya existía desde principios de 2016, no fue sino hasta el inicio del ciclo escolar 2016-1017 cuando el magisterio indígena tomó la decisión de aplicarlo.

EI PEPOMICH tiene su origen en los congresos pedagógicos que celebra la Dirección de Educación Indígena en Michoacán desde 1995; en estos espacios de discusión, docentes indígenas comparten cada año experiencias sobre cómo fomentan el uso de la lengua originaria y la aplicación de conocimientos indígenas. Si bien estas experiencias no se han publicado, comprenden un acervo importante que ahora constituye el punto de partida para el diseño y la implementación de un modelo propio. A la par con estas experiencias, se tuvieron proyectos y propuestas alternativas tanto de la parte sindical como de la Dirección de Educación Indígena, 
pero no se habían podido coordinar (docente de preescolar, entrevista, 2017).

Es un logro importante lo que se ha realizado desde el ciclo escolar 2016-2017; al respecto, una profesora que trabaja en una supervisión de educación preescolar indígena comenta: "Va de la mano la parte sindical con la parte oficial, inclusive [para] los talleres que estamos haciendo aquí, citan la parte oficial y la parte sindical". Estos talleres se ofrecen al inicio del ciclo escolar y son los espacios donde el profesorado recibe capacitación para el trabajo con el PEPOMICH.

La síntesis de los elementos básicos de cada una de estas propuestas se formula a partir de los resultados de la investigación documental y las entrevistas a docentes de educación indígena y representantes sindicales involucrados en su diseño y aplicación. A continuación los resumimos considerando fundamentos político-pedagógicos, actores, diseño curricular, conocimientos indígenas, papel de la investigación, y relación entre comunidad y escuela.

\section{Fundamentos políticos-pedagógicos}

Todo proceso formativo tiene objetivos determinados que corresponden a la perspectiva histórica, social y política de quienes diseñan los contenidos y su tratamiento pedagógico. Cuando se trata de proyectos alternativos, los componentes políticos pretenden transformar el sistema político y educativo existente a través de la autodeterminación de los pueblos originarios.

El MII es un proyecto educativo con el objetivo de crear "una educación intercultural y bilingüe [...], abierta a la participación comunitaria y culturalmente pertinente" (Bertely, 2007, p. 27). En contraste con el concepto oficial de educación intercultural que aspira a crear una sociedad tolerante y respetuosa frente a diferencias culturales, el MII plantea que la interculturalidad no se limita al contacto entre sujetos culturalmente diferentes, sino que se trata de sujetos sociales en un determinado contexto histórico-social que implica relaciones asimétricas de poder (Dietz, 2017) y las reconocen para transformarlas.

Por lo tanto, este tipo de educación intercultural no se dirige hacia el futuro, sino que debe proporcionar a docentes y estudiantes contenidos y métodos apropiados para dar cuenta de estas relaciones de poder, fortalecerlos y prepararlos para su superación mediante una democracia activa y solidaria frente a las estructuras que hacen funcionar el sistema.

El trabajo con el MIl se dirige exclusivamente a la población indígena para "abonar una educación ética y ciudadana intercultural y bilingüe fundada en una concepción pragmática, situada y activa de la cultura, explicitada a partir de las experiencias, las prácticas y los actos realizados en la vida diaria de los comuneros y en los territorios propios" (Bertely, 2007, pp. 31-32). De esta manera se recupera, en términos educativos escolares, la educación en y para el trabajo como esencia de la educación comunitaria. Su enfoque pedagógico está basado en Vygotski (zona de desarrollo próximo) y Leontiev (actividad y acción), cuyas aportaciones teóricas recuperó y desarrolló Jorge Gasché (2008) para el diseño del MII.

El caso del PEPOMICH es distinto, surge por un reclamo histórico que deriva de la necesidad de un plan y programas específicos que atiendan todos los niveles educativos de los pueblos originarios, desde la educación inicial hasta la superior. La inconformidad con los programas homogéneos en el ámbito nacional se dirige hacia los contenidos, la metodología pedagógica/didáctica y el uso de las lenguas originarias. Para dar respuesta a esta demanda, hay que considerar 
las necesidades y aspiraciones de los pueblos originarios, la recuperación de sus saberes, la cosmovisión y la vida comunal, para convertirlos en contenidos educativos, siendo necesario transformar el modelo educativo desde la selección de contenidos, la elección de enfoques, la práctica docente y el diseño de estrategias pedagógicas (DGEI y Sector IX, 2016, p. 22).

En concordancia con una educación autodeterminada, la metodología pedagógica articula prácticas pedagógicas de los pueblos originarios con las teorías de Freire, Bruner y Vygotski.

El programa está basado en los derechos educativos garantizados tanto por sustentos legales nacionales como internacionales; entre los primeros están la Constitución Política de los Estados Unidos Mexicanos, la Ley General de Derechos Lingüísticos y la Ley General de Educación; entre los segundos, el Convenio 169 de la Organización Internacional del Trabajo y también se fundamenta en los resolutivos de los congresos político-sindicales de la Coordinadora Nacional de Trabajadores de la Educación (CNTE).

Como puede observarse, salir de los esquemas o estereotipos que enmarcan el pensamiento occidental será una tarea ardua.

\section{Los actores}

A fin de promover la autodeterminación se requiere que quienes diseñan los proyectos y quienes participan en los procesos educativos sean las mismas personas; es decir, que no se mantenga la división tradicional entre diseñadores y ejecutores de los planes y programas educativos.

La propuesta del MII parte "desde abajo y desde adentro" (Bertely, 2006), desde los actores directos: los docentes de educación indígena en colaboración con los padres de familia y otros miembros de las comunidades. El diseño de actividades y la práctica educativa se encuentran en constante actualización para adecuarse a las condiciones y necesidades locales. La colaboración y co-teorización (Sartorello, 2014) entre indígenas y no indígenas es expresión de la educación intercultural e inductiva que caracteriza el MII.

Por su parte, en el PEPOMICH, desde su origen, están involucrados como agentes del cambio el magisterio, las autoridades educativas estatales, las autoridades civiles y comunales de las comunidades, las madres y los padres de familia y alumnos, "porque tenemos que tener la autorización de ellas para poder nosotros trabajar" (docente de educación inicial, entrevista, 2017). Durante la etapa de investigación y sistematización de los conocimientos locales se formaron equipos entre docentes, directivos, asesores técnico-pedagógicos y supervisores junto con representantes de la parte sindical. "Toda la información ha salido de la base, de las maestras que están frente a grupo [...] para ver qué material sirve y de qué manera lo pueden mejorar" (asesora técnica-pedagógica, entrevista, 2017). La coordinación general se comparte entre personal de la Dirección de Educación Indígena, la Escuela Normal Indígena de Michoacán y el Sector IX de la Sección XVIII de la CNTE-SNTE (Sindicato Nacional de Trabajadores de la Educación).

En cuanto a los actores, es innegable la participación de todos en ambas propuestas, lo que garantiza procesos democráticos y la transformación de las relaciones desiguales del poder. Ambas experiencias son ejemplos de la redistribución y 
resignificación del poder como posibilidad.

\section{Diseño curricular y conocimientos indígenas}

El diseño curricular es el componente sustantivo de cada plan y programa educativo. En él se sientan las bases de los contenidos, métodos y actividades sugeridas para los procesos de aprendizaje y enseñanza.

En el MII, el diseño curricular recupera las ideas y actividades de comuneras y comuneros indígenas; así, integra sociedad y naturaleza y no separa campos de conocimientos y disciplinas científicas, sino que "todos los conocimientos (comunitarios, escolares y universales) se desprenden de una sola área integradora, llamada Sociedad y Naturaleza" (UNEM et al., 2009, p. 83).

Con base en esta premisa de mantener la unidad entre sociedad y naturaleza, las actividades didácticas parten de los conocimientos indígenas que están implícitos en las "actividades sociales, productivas, rituales y recreativas que las personas indígenas realizan en su vida cotidiana" (UNEM et al., 2009, p. 84) y festiva. Los docentes hacen explícitos dichos conocimientos con base en sus investigaciones y preparan tanto las actividades como los materiales didácticos para ser trabajados dentro de la escuela o la comunidad junto con personas mayores, especialistas en determinadas habilidades y conocimientos.

El trabajo educativo que se lleva a cabo con el MII tiene como finalidad potenciar los conocimientos locales y de los pueblos originarios mediante su articulación con conocimientos escolares y científicos "universales" para fortalecer las comunidades indígenas en su forma de ser, hacer y darle sentido a la vida, contra el dominio neoliberal tanto económico y político como cultural.

El currículo está basado en la frase generadora "nosotros vamos a nuestro territorio a pedir un recurso que trabajamos para satisfacer nuestras necesidades sociales" (UNEM et al., 2009, p. 85) y contiene cuatro ejes temáticos: territorio-naturaleza; recurso natural-producto; trabajo-técnica; fin social-sociedad. Cada uno de estos ejes se subdivide en diferentes niveles de variables que permiten la articulación entre los ejes.

El MII busca que los niños construyan nuevos conocimientos a partir de lo que ya saben mediante un proceso inductivo que los acerca y habilita para el encuentro y la confrontación con otras culturas sin tener que renunciar a lo propio, sino, al contrario, para fortalecerlo.

Por otra parte, en el PEPOMICH, tanto el diseño como el desarrollo curricular están pensados en cinco etapas: planeación, instrumentación, ejecución, evaluación y un replanteamiento. Las etapas no están separadas; se conciben como ciclos que se llevan simultáneamente en pares durante algún tiempo, y la de replanteamiento es constante. Esto significa que el diseño está abierto a críticas y cambios a lo largo de su implementación.

Los ejes transversales que dan sustento a las líneas de trabajo son: la "cosmovisión propia de los pueblos originarios y comunidades indígenas" (DGEI y Sector IX, 2016, p. 69), el enfoque de comunalidad, una perspectiva descolonizadora, y la lengua y cultura originaria; sin dejar fuera la cultura y los conocimientos que proponen los programas nacionales, el bilingüismo o plurilingüismo, según las necesidades de la población.

Definimos siete líneas que se trabajan en todos los niveles educativos, desde inicial hasta superior: 
- La línea de "Vida y naturaleza" refleja la unidad entre humanos y naturaleza, e incluye las condiciones geográficas y biológicas desde una perspectiva de unidad interdependiente.

-En "Cosmovisión y valores" se relacionan la visión del mundo con los conocimientos, creencias y maneras de convivir.

- Cuando trabajan la línea "Tecnología tradicional" se abarcan conocimientos ubicados en los procesos productivos, de construcción, artes y oficios en general.

- "Historia y territorio" subraya la interrelación inseparable entre tiempo y espacio que ha forjado las identidades de los pueblos originarios.

- La línea de "Etnomatemáticas" se entiende como parte de la vida cotidiana; se aprende en la interacción social y está ligada a la cultura de cada pueblo.

- Los conocimientos implícitos que comprenden "la prevención, diagnóstico o tratamiento de enfermedades físicas, mentales y espirituales" (DGEI y Sector IX, 2016, p. 105) se tocan en la línea de "Medicina tradicional".

-En la línea de "Lengua" se profundiza en la estructura lingüística y la práctica como medio de comunicación social y expresión de la cultura y las artes en general. En aquellas comunidades donde ha desaparecido la lengua originaria se aplican estrategias de vitalización y rescate.

En ambos diseños es posible observar la resignificación de los elementos culturales de las comunidades indígenas; esto implica una lucha permanente que confronta distintos mundos. Esta confrontación se suscita en el currículo oculto, ese que se construye día a día en las aulas y está determinado por el inconsciente de cada actor; de ahí la importancia de la formación docente.

\section{Papel de la investigación}

La sistematización de los conocimientos indígenas solo es posible a través de la investigación in situ de las prácticas productivas, sociales, rituales y de diversión, es decir, en las comunidades donde se mantienen, reconstruyen y transmiten.

Cada comunidad tiene determinados conocimientos y realiza actividades específicas; por lo anterior, se sugiere que el profesorado inicie su trabajo en el MII con investigaciones sobre las condiciones de su propio contexto, cuyo propósito es dar cuenta de estas características. Desde los diplomados, quienes participan se forman con una perspectiva etnográfica en técnicas de observación y entrevista que les permiten re-conocer lo que se hace y piensa en la comunidad. En las temáticas que investigan centran la atención en las responsabilidades y los conocimientos de la infancia para potenciarlos mediante las actividades escolares. En este sentido, los resultados de la investigación contextual constituyen las bases indispensables para la planeación de actividades y el diseño de materiales educativos.

En el mismo sentido, como parte de la estructura del PEPOMICH, existe un equipo específico dedicado a la elaboración de material didáctico. Estos especialistas requieren las investigaciones que el profesorado lleva a cabo en las comunidades. El momento más intenso en la elaboración de materiales fue al inicio del proyecto, pero se ha dado un proceso continuo y de vinculación entre recuperación de datos tanto cuantitativos como cualitativos y diseño de materiales para complementar 
y actualizar los primeros resultados; de esta manera ha sido posible seguir con la elaboración de recursos didácticos.

En función de lo anterior, se reconoce la investigación como actividad central en el proceso de recuperación, generación y aplicación de conocimientos.

\section{Relación entre comunidad y escuela}

El MII tiene un procedimiento inductivo que lo caracteriza; por ello, la comunidad se considera el fundamento de los procesos educativos, tanto escolares como comunitarios. Las y los educadores deben participar en las actividades diversas de la comunidad para identificar los conocimientos indígenas implícitos y articularlos con los contenidos escolares. Al respecto, una maestra de primaria reconoce que "las conversaciones con las madres y los padres de familia me permitieron conocer y abundar más en los conocimientos y los significados locales. Admito que ante ellos era un aprendiz" (en Keyser, Silva, Hernández y Bertely, 2012, p. 10). Con este tipo de experiencias, quienes participan reconocen paulatinamente que la democracia activa y solidaria requiere relaciones horizontales entre los docentes, padres de familia y comuneros.

Por otra parte, desde el principio de comunalidad que atraviesa el desarrollo curricular del PEPOMICH, este le otorga importancia a la relación entre comunidad y escuela. Aquí la comunidad y sus pobladores son la fuente de conocimientos, de prácticas pedagógicas y el espacio reconocido donde tiene que dar fruto la educación escolar.

Las madres de familia apoyan la iniciativa de implementar juegos tradicionales y revalorar el trabajo en el campo porque, según su mirada, "los niños ya no quieren participar en los trabajos de la familia" (madre de familia, entrevista, 2017). Esto lleva a las docentes a "volver a integrar nuevamente a los niños al trabajo o que vayan rescatando lo que se está perdiendo" (directora y docente de preescolar, entrevista, 2017). En este sentido, no se trata solo de llevar la escuela a la comunidad y al campo para incorporar al trabajo escolarizado lo que ahí se vive y observa, sino que la escuela ahora tiene el papel de rescatar el pasado de las comunidades y que se ha perdido por el proceso de escolarización, por ejemplo, la lengua originaria en muchas poblaciones indígenas y el trabajo entre familiares en el campo.

Con estos planteamientos y actividades se pretende romper la dicotomía de dos espacios distintos que representen la comunidad y la escuela. Cuando ambas instancias se reconocen, una se integra en la otra y se desdibujan sus límites.

\section{CONCLUSIONES}

Al principio de este escrito planteamos dos interrogantes relacionadas con la manera en que se reconocen los conocimientos indígenas dentro de programas educativos oficiales y alternativas para analizar estas propuestas como posibilidades que contribuyan al empoderamiento de los saberes indígenas.

Al momento de revisar los programas mencionados emergieron otros aspectos que merecían ser analizados por ser parte de la formación y práctica de docentes que laboran en contextos de pueblos originarios. Tal es el caso de los fundamentos político-pedagógicos de dichos programas, porque los tres refieren un sector social vulnerable de la nación mexicana y pueden ser parte de un proyecto político que permita superar esa condición de vulnerabilidad. 
El análisis de las propuestas destaca la relevancia de la formación de docentes para consolidar propuestas alternativas orientadas a la emancipación y reconoce como un primer paso el ejercicio que se tiene en la LEPEPMI. Esta se centra en asuntos de la lengua y la cultura y, con base en esto, en una resignificación identitaria como indígenas; sin embargo, estos temas resultan insuficientes para constituirse en alternativas que permitan transformar tanto las estructuras económicas y políticas como el sistema educativo nacional, que son la base de las relaciones asimétricas de poder.

Tanto el MII y como el PEPOMICH se conciben a partir de una postura política de democracia activa y solidaria, de respeto a las diferencias culturales y lingüísticas, los conocimientos tradicionales locales, así como las experiencias y los saberes de la infancia. Ambas propuestas están diseñadas para el trabajo con la población indígena y la articulación entre conocimientos de distinto origen cultural desde "abajo" y "adentro" de las mismas comunidades. No se trata de incluir conocimientos indígenas en la escuela, sino de potenciar los conocimientos locales mediante su articulación con conocimientos escolares y científicos occidentales para fortalecer las comunidades indígenas en sus formas de ser y hacer, con un ethos propio que permita dar otro sentido a la vida, opuesto al que implica el dominio neoliberal económico, político y cultural.

Como es sabido en estas relaciones asimétricas de poder, si subyacen elementos que refieren una violencia estructural y cultural -imperceptibles por ser aceptadas como situaciones normales relacionadas con un deber ser-, tendría que ser prioridad de los formadores de docentes atender los procesos que impiden al docente reflexionar, analizar y dar sentido a su práctica más allá de la reproducción cultural.

En este sentido, un planteamiento curricular alternativo se propone "una educación que está explícita y deliberadamente orientada a revertir el modelo social excluyente desde el que ha sido configurada la sociedad" (Méndez, citado en Bermúdez, Cuevas y Hernández, 2015, p. 364) y, para lograrlo, habrían de crearse nuevas formas de relaciones económicas e ideológicas distintas a las que dominan y que fueron creadas para facilitar el desarrollo del capitalismo.

Una vez aclarados los fundamentos político-pedagógicos, debemos preguntarnos por los actores como diseñadores y ejecutores de los programas que hacen posible su concreción dentro de la vida escolar y comunitaria. En las alternativas que se analizaron para este trabajo encontramos que los mismos docentes participan como diseñadores y que esta condición posibilita la constante revisión y, si fuera necesario, actualización de los programas. A la vez, el cambio del estatus del docente de ser solo ejecutor a ser creador de los fundamentos teórico-metodológicos de su trabajo, lo empodera para superar el estado de cierta enajenación entre el currículo formal y el real.

El diseño curricular, como categoría de análisis preestablecida desde un inicio, junto con la de conocimientos indígenas, constituye uno de los aspectos centrales de nuestra investigación. Si los conocimientos indígenas no obedecen a una división en campos disciplinarios de acuerdo con la ciencia dominante, las áreas de formación y práctica docente tendrían que ser otras. Mientras que en la LEPEPMI se sigue reflejando la división por campos disciplinarios, en el PEPOMICH, a excepción de "Etnomatemáticas", "Medicina tradicional" y "Lengua”, se superó esta división y dio lugar a espacios de conocimiento que reflejan una aproximación epistemológica más integral. En el caso del MII y en concordancia con su perspectiva ontológica- 
epistemológica de la integridad entre sociedad y naturaleza, cada eje de formación la refleja, sin hacer alusión a campos disciplinares.

En los tres programas revisados se registra la recuperación de saberes y los conocimientos locales, además de su reconstrucción y aplicación para la solución de problemas comunitarios. Sin embargo, solo en los programas alternativos se hace referencia a la participación de todos los sectores implicados, al visualizar el aprendizaje en relaciones igualitarias dentro y fuera de las instituciones educativas.

El programa de licenciatura, al pertenecer al nivel de educación superior, requiere una investigación especializada como parte del proceso formativo del profesorado. A la vez, el acento en el re-conocimiento de saberes y conocimientos indígenas la hace indispensable. Por ello, los programas alternativos la consideran parte esencial en la capacitación y constante en práctica docente. El trabajo con las propuestas alternativas demanda procesos de investigación y diagnóstico; es decir, el profesorado tiene que buscar las estrategias idóneas para apropiarse de los conocimientos y las habilidades que caracterizan la vida en el contexto sociocultural de sus estudiantes. Tanto en el PEPOMICH como en el MII se considera indispensable la participación de docentes en la vida comunitaria para promover aprendizajes significativos y educar con sentido democrático y emancipatorio.

Superar la división entre principios educativos y estructuras asimétricas de poder, o entre escuela y comunidad, es parte de los objetivos de los programas alternativos. Podemos concluir que el primer programa universitario específico para la formación de docentes indígenas cimentó las bases para crear nuevos espacios que permitan cuestionar y construir alternativas con una educación intercultural que fortalezca el yo indígena sin disolverlo en el Otro.

\section{REFERENCIAS BIBLIOGRÁFICAS}

Bermúdez, F., Cuevas, J. y Hernández, A. (2015). Experiencias educativas dentro del sistema educativo oficial mexicano. ¿Es posible ser alternativo desde la institución oficial? En P. Medina (coord.). Pedagogías insumisas. Movimientos político-pedagógicos en memorias colectivas de educaciones otras en América Latina (pp. 363-379). México: Centro de Estudios Superiores de México y Centroamérica/Juan Pablos.

Bertely Busquets, M. (2007). Conflicto intercultural, educación y democracia en México. Ciudadanía y derechos indígenas en el movimiento pedagógico intercultural bilingüe en los Altos, la Región Norte y la Selva Lacandona de Chiapas. México: CIESAS.

Bertely Busquets, M. (2006). La construcción desde debajo de una nueva educación intercultural bilingüe para México. En E. Todd y V. Arredondo (coords.). La educación que México necesita. Visión de expertos (pp. 29-41). México: Centro de Altos Estudios e Investigación Pedagógica/CECyTE.

Bertely, M. (coord.) y REDIIN (2011). Interaprendizajes entre indígenas, de cómo las y los educadores pescan conocimientos y significados comunitarios en contextos interculturales. México: CIESAS.

Castro-Gómez, S. (2015). Revoluciones sin sujeto. México: Akal.

Dietz, G. (2017). Interculturalidad: una aproximación antropológica. Perfiles Educativos, vol. 39, núm. 156, pp. 192-207. Recuperado de http://www.iisue.unam.mx/ perfiles/articulo/2017-156-interculturalidad-una-aproximacion-antropologica. pdfDietz, G. y Mateos, L. S. (2011). Interculturalidad y educación intercultural en México: un análisis de los discursos nacionales e internacionales en su impacto en los modelos educativos mexicanos. México: SEP/CGEIB. 
Dirección de Educación Indígena en el Estado de Michoacán (2017). Datos estadísticos de educación primaria indígena. Documento interno. Morelia, Michoacán: Secretaría de Educación en el Estado.

Dirección General de Educación Indígena y Sector IX de la Sección XVIII, CNTE-SNTE (2016). Proyecto para el Desarrollo Curricular Alternativo de Educación Indígena en Michoacán. Morelia, Michoacán: Dirección General de Educación Indígena.

Foucault, M. (2014). Vigilar y castigar. México: Siglo XXI.

Galtung, J. (2003). Violencia cultural. Recuperado de gernikagogoratuz.org: https:// www.gernikagogoratuz.org/web/uploads/documentos/202892edd66aafe5c03da cf1298fd7f8938fae76.pdf

Gasché, J. (2008). Niños, maestros, comuneros y escritos antropológicos como fuentes de contenidos indígenas escolares y la actividad como punto de partida de los procesos pedagógicos interculturales: un modelo sintáctico de cultura. En M. Bertely Busquets, J. Gasché y R. Podestá (coords.). Educando en la diversidad. Investigaciones y experiencias educativas interculturales y bilingües (pp. 279-365). México: CIESAS/Quito, Ecuador: Abya-Yala.

Gramsci, A. (2013). Cuadernos de la cárcel. Los intelectuales y la organización de la cultura. México: Juan Pablós.

Il Cumbre Continental de los Pueblos y Nacionalidades Indígenas de AbyaYala (2004). Tema 4: Conocimientos indígenas y propiedad intelectual. Quito, Ecuador. Recuperado de http://www.cumbreindigenabyayala.org/ponencias/tema4.html

Keyser, U. (2011). Procesos de cambio en la formación de docentes indígenas al practicar educación intercultural en la región purhépecha de Michoacán. En M. Bertely (coord.) y REDIIN. Interaprendizajes entre indígenas, de cómo las y los educadores pescan conocimientos y significados comunitarios en contextos interculturales (pp. 117-149). México: CIESAS.

Keyser, U., Silva, E., Hernández, M. y Bertely, M. (2012). Guía para sembrar el método inductivo intercultural en comunidades y escuelas. Red de Educación Inductiva Intercultural (REDIIN), Michoacán, Pueblo P'urhépecha. México: CIESAS.

Lacan, J. (2016). Escritos I. Recuperado de Bibliopsi.org: http://www.bibliopsi.org/ docs/lacan/33\%20Los\%20Escritos\%20de\%20Jacques\%20Lacan.pdf

Pérez, M. L. y Argueta, A. (2011). Saberes indígenas y diálogo intercultural. Cultura y Representaciones Sociales, vol. 5, núm. 10, pp. 31-56.

Quilaqueo, D. (2007). Saberes y conocimientos indígenas en la formación de profesores de educación intercultural. Educar em Revista, núm. 29, pp. 223-239. Recuperado de http://www.redalyc.org/articulo.oa?id=155013355015

Reyes, S. (2016). La problemática de la formación docente. Caso LEP y LEPMI 90. Educación y Ciencia, vol. 5, núm. 45, pp. 106-120. Recuperado de http://www.educacionyciencia.org/index.php/educacionyciencia/article/view/374/pdf_29

Sartorello, E. (2014). La co-teorización intercultural de un modelo curricular en Chiapas, México. Revista Mexicana de Investigación Educativa, vol. 19, núm. 60, pp. 73-101. Recuperado de http://www.redalyc.org/pdf/140/14029405005.pdf

UNEM/ECIDEA/CIESAS/IIAP/OEI (2009). Modelo curricular de educación intercultural bilingüe. México: CIESAS.

UPN (s.f). Plan de Estudios de la Licenciatura en Educación Preescolar y Primaria para el Medio Indígena, LEPEMI '90. Recuperado de http://www.upnmda.edu.mx/attachments/article/87/plan_estudios_lepepmi.pdf 
UPN (2016a). Documento normativo LEPEPMI. Recuperado de www.upnhidalgo.edu. $\mathrm{mx} /$ textos/.../Documento\%20Normativo\%20LEPEPMI'90.pdf

UPN (2016b). Guías del estudiante LEPEPMI. Recuperado de http://200.23.113.51/ pdf/guias/

UPN (2002). Guías de estudio de la LEP y LEPMI. Recuperado de http://biblioteca. ajusco.upn.mx/web/guias-de-estudio.php

Villoro, L. (2008). Creer, saber, conocer. México: Siglo XXI.

Von Groll, M., Keyser Ohrt, U. y Silva Castellón, E. (2013). Formación de maestros indígenas y no indígenas para la educación indígena e intercultural. En M. Bertely Busquets, G. Dietz y M. G. Díaz Tepepa (coords.). Multiculturalismo y educación, 2002-2011 (pp. 151-183). México: ANUIES/COMIE.

Walsh, C. (2006). Interculturalidad, conocimientos y (de) colonialidad. En CEFIA-UICCGEIB (coord.). Política e interculturalidad en educación. Memoria del II Encuentro Multidisciplinario de Educación Intercultural (pp. 17-29). México: CGEIB. 